Poincare Journal of Analysis E Applications

Vol. 2015 (2), Special Issue (IWWFA-II, Delhi), 77-92

CPoincare Publishers

\title{
MATRIX APPROACH TO THE GLOBAL AND LOCAL REGULARITY OF WAVELETS
}

\author{
Vladimir Yu. Protasov ${ }^{\dagger}$ and Nicola Guglielmi \\ $\begin{array}{lll}\text { Date of Receiving } & : & 20.05 .2015 \\ \text { Date of Revision } & : & 16.11 .2015 \\ \text { Date of Acceptance } & : & 16.11 .2015\end{array}$
}

\begin{abstract}
We apply recent methods of computing the joint spectral characteristics of matrices to find precise values of the Hölder exponents of Daubechies wavelets of order $N \leq 20$. The local regularity of these wavelets is also analyzed. In particular, we find the points of the worst local regularity. In all cases they are rational, but not always dyadic.
\end{abstract}

\section{Introduction}

The regularity of wavelets and of refinement equations is one of key characteristics, important for their approximation properties, for applications in PDE, in functional analysis etc. This problem was studied in detail, see $[8,9,7,12,11,19,32,35,36]$ and references therein. Among several methods of estimating the regularity of wavelets elaborated in the literature the matrix approach is the only one that gives (at least theoretically) precise values of the Hölder exponent. Moreover, in some cases it allows us to make a refine analysis of local regularity of wavelet functions. The main disadvantage is that the matrix approach reduces the problem of finding the Hölder exponent to computing of the joint spectral characteristics of matrices. This problem is notoriously hard even for matrices of low dimensions. That is why the exponents of regularity was computed only for wavelets with small support. Recently several efficient methods of computing the joint spectral characteristics was elaborated. In this paper we apply one of them, the invariant polytope algorithm, to find precise values of the Hölder exponent of compactly supported wavelets. In particular, we find the regularity of Daubechies wavelets of order $N \leq 20$. We disprove the conjecture that the

2010 Mathematics Subject Classification. 15A60, 15-04, 15A18, 90C90.

Key words and phrases. wavelets, Hölder exponent, joint spectral radius, invariant polytope algorithm, dominant product.

The first author is supported by RFBR grants nos. 13-01-00642 and 14-01-00332, and by the grant of Dynasty foundation. The second author is supported by INdAM GNCS (Gruppo Nazionale di Calcolo Scientifico). The authors are very grateful to the anonymous referee for attentive reading and remarks. A part of this work has been done during the visit of V.P. at the University of L'Aquila and GSSI (L'Aquila, Italy). He is grateful for their hospitality.

Communicated by. Yuri A. Farkov

${ }^{\dagger}$ Corresponding author. 